\title{
Residual resultant over the projective plane and the implicitization problem
}

\author{
Laurent Busé \\ UNSA, UMR 6621 / INRIA, GALAAD \\ Parc Valrose, B.P. 71, 06108 Nice, France \\ lbuse@math.unice.fr
}

\begin{abstract}
In this article, we first generalize the recent notion of residual resultant of a complete intersection the case of a local complete intersection of codimension 2 in the projective plane, which is the necessary and sufficient condition for a system of three polynomials to have a solution "outside" a variety, defined here by a local complete intersection of codimension 2. We give its degree in the coefficients of each polynomial and compute it as the gcd of three polynomials or as a product of two determinants divided by another one. In a second part we use this new type of resultant to give a new method to compute the implicit equation of a rational surface with base points in the case where these base points are a local complete intersection of codimension 2 .
\end{abstract}

\section{INTRODUCTION}

The implicitization problem asks for the implicit equation of a parametrized surface. The parametrization of such a surface is usually defined by the equations

$$
\frac{q_{1}\left(x_{1}, x_{2}\right)}{q_{4}\left(x_{1}, x_{2}\right)}, \frac{q_{2}\left(x_{1}, x_{2}\right)}{q_{4}\left(x_{1}, x_{2}\right)}, \frac{q_{3}\left(x_{1}, x_{2}\right)}{q_{4}\left(x_{1}, x_{2}\right)},
$$

where $q_{1}\left(x_{1}, x_{2}\right), q_{2}\left(x_{1}, x_{2}\right), q_{3}\left(x_{1}, x_{2}\right)$ and $q_{4}\left(x_{1}, x_{2}\right)$ are four polynomials in two variables. If we homogenize these four polynomials, we obtain the general case : let

$$
\theta: \mathbb{P}^{2} \longrightarrow \mathbb{P}^{3}:\left(x_{0}: x_{1}: x_{2}\right) \mapsto\left(p_{0}: p_{1}: p_{2}: p_{3}\right)
$$

be a rational map (i.e. polynomials $p_{0}, p_{1}, p_{2}, p_{3}$ can vanish simultaneously at some points) given by four homogeneous polynomials $p_{0}, p_{1}, p_{2}, p_{3}$ of the same degree $d$, and whose image is a surface $S$ of $\mathbb{P}^{3}$. The implicitization problem consists in computing the implicit equation of $S$, that is the smallest algebraic surface of $\mathbb{P}^{3}$ containing $S$. Computing the implicit equation of a parametric surface is a key point in computer-aided design and in many problems of computational geometry. For instance, it helps drawing a parametric surface nearby a singularity, computing autointersection of offsets and drafts, or computing the intersection with curves or surfaces (see [6]). The degree of the implicit equation is known to be $d^{2}$ when there are no base points (a base point is a point of $\mathbb{P}^{2}$ where polynomials $p_{0}, p_{1}, p_{2}, p_{3}$ vanish simultaneously), and less when there exists base points. In practice the map $\theta$ has very often base points which appear naturally either for geometrical or design reasons. As an illustration,

ISSAC 2001, UWO, Canada a classical result says that a generic cubic contains 27 lines which are closely related to the cubic parametrization with 6 base points (see [16]).

There are three known methods to solve the implicitization problem and all of them reduce the problem to the elimination of variables. The first method is based on Gröbner basis computations. In the abscence of base points, a Gröbner base computation of the ideal $I$ generated by the polynomials $p_{0}, p_{1}, p_{2}, p_{3}$ gives the implicit equation. If there exist base points, the implicit equation does not belong to the ideal $I$ but to the saturated ideal of $I$ by the ideal defining the base points. So, in this case, we need to compute this saturated ideal and a Gröbner base of it to obtain the implicit equation. This method can be very slow in practice (see [21], [0]).

The second method for computing the implicit equation consists in computing a classical projective resultant of the polynomials $p_{0}-x p_{3}, p_{1}-y p_{3}, p_{2}-z p_{3}$ (see [10], 11]), but this method failed in the presence of base points. In 8 and 5] perturbation techniques are used to obtain the implicit equation by a computation of a classical resultant in the presence of base points. In fact the implicit equation is contained in the lowest degree term of the resultant of the perturbed system (expressed in terms of the perturbing variable) and we need to compute the gcd of $d+1$ polynomials to erase the extraneous factor (with some technical conditions). Notice also that it is always possible to compute a multiple of the implicit equation by computing the determinant of a maximal minor of the Bezoutian matrix of $p_{0}-x p_{3}, p_{1}-y p_{3}, p_{2}-z p_{3}$ (see [3]), but here we are interested in finding exactly the implicit equation.

The third method to solve the implicitization problem is the method introduced by Tom Sederberg in [24 and called the moving quadric method. This method is based on the study of the syzygies of the ideal $I$ defined by the polynomials $p_{0}, p_{1}, p_{2}, p_{3}$ and gives the implicit equation as a determinant of a certain matrix. The formulation of the implicit equation in a determinantal form can be very useful in practice. However, the validity of this method is only proved in the abscence of base points and in the case of tensor product surfaces (see [13], [14], 12]).

In this paper we will give a new method for computing the implicit equation of a rational surface with base points using resultants and syzygies. We will obtain the implicit equation as a resultant using the fact that the module of first syzygies of the ideal defining the base points locus (of codimension 2 in $\mathbb{P}^{2}$ ) is always free, whereas this is not true for the ideal $I$ (see [12]). In the recent paper [4, the notion of residual 
resultant of a complete intersection was introduced; it corresponds to the following situation : let $g_{1}, \ldots, g_{n}$ be homogeneous polynomials of degree $k_{1}, \ldots, k_{n}$ in $\mathbb{K}\left[x_{0}, \ldots, x_{m}\right]$ and $d_{0}, \ldots, d_{m}$ be $m+1$ positive integers. We suppose that the ideal $G=\left(g_{1}, \ldots, g_{n}\right)$ is a complete intersection. The residual resultant is the condition on the coefficients $\mathbf{c}=\left(c_{\alpha}^{i, j}\right)$ such that the system

$$
\left\{\begin{aligned}
f_{0}(x) & =\sum_{i=1}^{n} h_{i, 0}(x) g_{i}(x) \\
\vdots & \\
f_{m}(x) & =\sum_{i=1}^{n} h_{i, m}(x) g_{i}(x)
\end{aligned}\right.
$$

(where $h_{i, j}=\sum_{|\alpha|=d_{j}-k_{i}} c_{\alpha}^{i, j} x^{\alpha}$ is the generic homogeneous polynomial of degree $d_{j}-k_{i}$ ) has a solution "outside" the variety $V\left(g_{1}, \ldots, g_{n}\right)$. This residual resultant can be used to compute a resultant in the presence of base points whereas the classical and toric resultants are degenerated. We will use techniques from algebraic geometry and commutative algebra to extend this notion of residual resultant in a new context, the case where $G$ is a local complete intersection of codimension 2 in $\mathbb{P}^{2}$. This extension is very useful since, for instance, a set of points of multiplicity 1 in $\mathbb{P}^{2}$ is always a local complete intersection and almost never a complete intersection. We will give the degree of this new residual resultant in the coefficients of each polynomials and two algorithms to compute it. To do this we will proceed as in 41, in two steps : first we define the residual resultant as a general resultant (see [18], chapter 3) over a blow-up variety, then we will use a free resolution of the residual ideal to obtain the residual resultant as the determinant of a complex. Finally we will apply this new residual resultant to the implicitization problem. We will show that this residual resultant can be used to compute the implicit equation of a rational surface in the presence of base points which are locally given by a complete intersection (with some technical conditions). In that case we will obtain the implicit equation as the gcd of three polynomials and also as a product of two determinants divided by another one (or a ratio of two determinants in special cases).

Hereafter $\mathbb{K}$ denotes an algebraically closed field.

\section{RESIDUAL RESULTANT OVER $\mathbb{P}^{2}$}

In this section we want to extend the notion of residual resultant over a complete intersection introduced in 胞 to the case of a local complete intersection in $\mathbb{P}^{2}=\operatorname{Proj}\left(\mathbb{K}\left[x_{0}, x_{1}, x_{2}\right]\right)$. More precisely, let $g_{1}, \ldots, g_{n} \in R=\mathbb{K}\left[x_{0}, x_{1}, x_{2}\right]$ be $n$ homogeneous polynomials of respective degrees $k_{1}, \ldots, k_{n}$ and $d_{0}, d_{1}, d_{2}$ be 3 positive integers. We suppose that the ideal $G=\left(g_{1}, \ldots, g_{n}\right)$ of $R$ is a local complete intersection and we consider the following system :

$$
\left\{\begin{array}{l}
f_{0}(x)=\sum_{i=1}^{n} h_{i, 0}(x) g_{i}(x) \\
f_{1}(x)=\sum_{i=1}^{n} h_{i, 1}(x) g_{i}(x) \\
f_{2}(x)=\sum_{i=1}^{n} h_{i, 2}(x) g_{i}(x)
\end{array}\right.
$$

where $x \in \mathbb{P}^{2}$ and $h_{i, j}(x)=\sum_{|\alpha|=d_{j}-k_{i}} c_{\alpha}^{i, j} x^{\alpha}$ is a homogeneous polynomial of degree $d_{j}-k_{i}$ for $i=1, \ldots, n$ and $j=0,1,2$. The polynomials $f_{i}(x)$ for $i=0,1,2$, are homogeneous polynomials of degree $d_{i}$ in the ideal $G$ and the coefficients $\mathbf{c}=\left(c_{\alpha}^{i, j}\right)$ are called the parameters of the system. We are looking for a condition on the coefficients c such that the system (11) has a solution which is not in the algebraic variety $V(G)$ defined by the ideal $G$. We will call such a condition, when it exists, the residual resultant and we will give two algorithms to compute it explicitly.

Before going further into details, we remark that the codimension one part of the variety $V(G)$ can be removed by dividing the polynomials $f_{0}, f_{1}, f_{2}$ by the gcd of $g_{1}, \ldots, g_{n}$. So we will only consider the relevant case where $G$ is a local complete intersection of codimension 2 . In the following we will use techniques from algebraic geometry and hence its language. We refer to 20 and 19] for complete references. We recall that $\mathcal{O}_{X}$ is the sheaf of rings of regular functions over a variety (or a scheme) $X$ and that, if $\mathcal{L}$ is a sheaf on $X$, $H^{0}(X, \mathcal{L})$ denotes the zeroth cohomology group of $\mathcal{L}$, that is its global sections.

\subsection{The residual resultant}

Hereafter $X$ denotes the projective plane $\mathbb{P}^{2}=\operatorname{Proj}(R)$ and we suppose that $d_{0} \geq d_{1} \geq d_{2} \geq k_{1} \geq \cdots \geq k_{n}$. Let $\mathcal{G}$ be the coherent sheaf of ideals associated to $G$ which is supposed to be a local complete intersection of codimension 2. Denoting by $\mathcal{G}\left(d_{i}\right)=\mathcal{G} \otimes_{\mathcal{O}_{X}} \mathcal{O}_{X}\left(d_{i}\right)$ for $i=0,1,2$, the vector space $V_{i}=H^{0}\left(X, \mathcal{G}\left(d_{i}\right)\right)$ is the set of homogeneous polynomials of degree $d_{i}$ which are in the saturation of the ideal $G$. We denote also by $\pi: \tilde{X} \rightarrow X$ the blow-up of $X$ along the coherent sheaf of ideals $\mathcal{G}$ (see [20]).

Proposition 2.1. Suppose that $d_{2} \geq k_{n}+1$. Then there exists an irreducible polynomial on $\prod_{i=0}^{2} V_{i}$, denoted by $\operatorname{Res}_{\mathcal{G}, d_{0}, d_{1}, d_{2}}$, which satisfies

$$
\operatorname{Res}_{\mathcal{G}, d_{0}, d_{1}, d_{2}}\left(f_{0}, f_{1}, f_{2}\right)=0 \Longleftrightarrow F^{\text {sat }} \neq G^{\text {sat }},
$$

where $F=\left(f_{0}, f_{1}, f_{2}\right)$ and $F^{\text {sat }}$ (resp. $\left.G^{\text {sat }}\right)$ denote the saturation of the ideal $F$ (resp. $G$ ).

Proof. We denote by $\tilde{\mathcal{G}}=\pi^{-1} \mathcal{G} \cdot \mathcal{O}_{\tilde{X}}$ the inverse image sheaf of the sheaf $\mathcal{G}$ on $\tilde{X}$ and by $\tilde{\mathcal{G}}_{d_{i}}$ the invertible sheaf $\tilde{\mathcal{G}} \otimes \pi^{*}\left(\mathcal{O}_{X}\left(d_{i}\right)\right)$, for $i=0,1,2$. Now proposition 3 of [4] tells us that $\operatorname{Res}_{\mathcal{G}, d_{0}, d_{1}, d_{2}}$ exists and vanishes if and only if there exists a point $x \in \tilde{X}$ such that the global section $\pi^{*}\left(f_{i}\right)$ of $\tilde{\mathcal{G}}_{d_{i}}$ vanishes at $x$ simultaneously for all $i=0,1,2$. So it remains to show that this condition is equivalent to $F^{\text {sat }} \neq G^{\text {sat }}$.

First if we suppose that $F^{s a t}=G^{s a t}$ then ideals $F$ and $G$ define the same blow-up of $X$ and so $\operatorname{Res}_{\mathcal{G}, d_{0}, d_{1}, d_{2}}\left(f_{0}, f_{1}, f_{2}\right)$ does not vanish. Conversely, suppose that this residual resultant does not vanish. The sheaf $\mathcal{G}$ defines $p$ points $P_{i}$ for $i=1, \ldots, p$, and the exceptional divisor of the blow-up $\pi$ is composed of $p$ exceptional divisors $E_{i}$ above each point $P_{i}$. Since $G$ is a local complete intersection, the sheaf $\mathcal{G} / \mathcal{G}^{2}$ is locally free of rank 2 and we have $\tilde{\mathcal{G}} / \tilde{\mathcal{G}}^{2} \simeq \mathcal{O}_{E_{1}+\ldots+E_{p}}(1)$. Therefore if the residual resultant does not vanish, then $\pi^{*}\left(f_{0}\right), \pi^{*}\left(f_{1}\right)$ and $\pi^{*}\left(f_{2}\right)$ generate $\tilde{\mathcal{G}}$. We deduce that $G$ and $F$ have the same saturation.

Notice that the hypothesis $d_{2} \geq k_{n}+1$ is needed in propo-

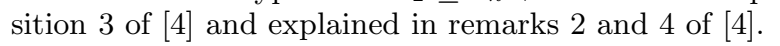

REMARK 2.2. $F^{\text {sat }} \neq G^{\text {sat }}$ is equivalent to $\left(F^{\text {sat }}: G^{\text {sat }}\right) \neq$ $R$, that is the variety defined by the ideal $(F: G)$ is not empty.

The ideal sheaf $\mathcal{G}$ defines a zero dimensional scheme $Z$ composed of $p$ points $P_{1}, \ldots, P_{p}$. We denote by $e_{i}$ the multiplicity of the point $P_{i}$ in $Z$. As $G$ is a local complete intersection 
we have $e_{i}=\operatorname{dim}_{\mathbb{K}}\left(\mathcal{O}_{Z, p}\right)$, where $\mathcal{O}_{Z}=\mathcal{O}_{\mathbb{P}^{2}} / \mathcal{G}$, and hence $\sum_{i=1 \ldots p} e_{i}=\operatorname{dim}_{\mathbb{K}} H^{0}\left(Z, \mathcal{O}_{Z}\right)$.

Proposition 2.3. The polynomial $\operatorname{Res}_{\mathcal{G}, d_{0}, d_{1}, d_{2}}$ is multihomogeneous in the coefficients of each $V_{i}$ of degree $N_{i}$ for $i=0,1,2$, with

$$
N_{i}=\frac{d_{0} d_{1} d_{2}}{d_{i}}-\operatorname{dim}_{\mathbb{K}} H^{0}\left(Z, \mathcal{O}_{Z}\right)=\frac{d_{0} d_{1} d_{2}}{d_{i}}-\sum_{i=1}^{p} e_{i} .
$$

Proof. We fix $i=0$. We have to compute the integer $N_{0}$ which, by propositions 1 and 3 of [4], equals

$$
\int_{\tilde{X}} c_{1}\left(\tilde{\mathcal{G}}_{d_{1}}\right) c_{1}\left(\tilde{\mathcal{G}}_{d_{2}}\right)
$$

where $c_{1}\left(\tilde{\mathcal{G}}_{d_{i}}\right)$ denotes the first chern class of the sheaf $\tilde{\mathcal{G}}_{d_{i}}$ and $\int_{\tilde{X}}$ the degree map on $\tilde{X}$. We denote by $H=\pi^{*}(h)$ where $h$ is the class of a generic hyperplane of $\mathbb{P}^{2}$, by $E_{i}$ for $i=, 1 \ldots, p$ the exceptional divisors of the blow-up $\pi$ above each point $P_{i}$ defined by $\mathcal{G}$, and by $E_{i}^{\text {red }}$ the reduced scheme of $E_{i}$. According to 17, $c_{1}\left(\tilde{\mathcal{G}}_{d_{i}}\right)=d_{i} H-\sum_{i=1}^{p} E_{i}$. Since $E_{i} . E_{j}=0$ if $i \neq j$ and $H . E_{i}=0$ for all $i=1, \ldots, p$, we deduce that

$$
N_{0}=\int_{\tilde{X}} d_{1} d_{2} H^{2}+\sum_{i=1}^{p} E_{i}^{2} .
$$

Now, let $f_{1}$ (resp. $f_{2}$ ) be a generic global section of $\mathcal{G}\left(d_{1}\right)$ $\left(\right.$ resp. $\left.\mathcal{G}\left(d_{2}\right)\right)$. We have

$$
\pi^{*}\left(f_{1}\right)=\tilde{f}_{1}+\sum_{i=1}^{p} E_{i}=\tilde{f}_{1}+\sum_{i=1}^{p} m_{i} E_{i}^{r e d},
$$

and

$$
\pi^{*}\left(f_{2}\right)=\tilde{f}_{2}+\sum_{i=1}^{p} E_{i}=\tilde{f}_{2}+\sum_{i=1}^{p} n_{i} E_{i}^{r e d},
$$

where $\tilde{f}_{1}$ (resp. $\tilde{f}_{2}$ ) is the strict transform of $f_{1}$ (resp. $f_{2}$ ), and where $m_{i}$ (resp. $n_{i}$ ) is the multiplicity of $f_{1}$ (resp. $f_{2}$ ) at the point $P_{i}$, that is, denoting by $\mathfrak{m}$ the maximal ideal of $R$ defining the point $P_{i}$, the greatest integer $s$ such that $f_{1}$ (resp. $f_{2}$ ) is in $\mathfrak{m}^{s}$.

Now $\tilde{f}_{1} \cdot \tilde{f}_{2}=0$ and since $\mathcal{G}$ is a local complete intersection we have $m_{i} n_{i}=e_{i}$. We deduce that

$$
\sum_{i=1}^{p} E_{i}^{2}=\sum_{i=1}^{p} m_{i} n_{i} E_{i}^{r e d^{2}}=\sum_{i=1}^{p} e_{i} E_{i}^{r e d^{2}} .
$$

By the projection formulae we know that $\int_{\tilde{X}} H^{2}=1$ and for all $i=1, \ldots, p, \int_{\tilde{X}} E_{i}^{r e d}{ }^{2}=-1$. We deduce that

$$
N_{0}=\int_{\tilde{X}} d_{1} d_{2} H^{2}+\sum_{i=1}^{p} e_{i} E_{i}^{r e d^{2}}=d_{1} d_{2}-\sum_{i=1}^{p} e_{i} .
$$

If we suppose moreover that $G$ is a saturated ideal we can give a more explicit result for the degrees $N_{0}, N_{1}$ and $N_{2}$. The Hilbert-Burch theorem tells us that there exists a minimal graded resolution of $R$-modules of the ideal $G$ of the form (see [15], theorem 20.15) :

$$
0 \rightarrow \bigoplus_{i=1}^{n-1} R\left[-l_{i}\right] \stackrel{\psi}{\longrightarrow} \bigoplus_{i=1}^{n} R\left[-k_{i}\right] \stackrel{\gamma=\left(g_{1}, \ldots, g_{n}\right)}{\longrightarrow} G \rightarrow 0
$$

with $\sum_{i=1}^{n-1} l_{i}=\sum_{i=1}^{n} k_{i}$.

Proposition 2.4. Suppose that $G$ is a saturated ideal with minimal resolution (2), then for $i=0,1,2$, we have

$$
N_{i}=\frac{d_{0} d_{1} d_{2}}{d_{i}}-\frac{\sum_{j=1}^{n-1} l_{j}^{2}-\sum_{j=1}^{n} k_{j}^{2}}{2} .
$$

Proof. We have an exact complex of sheaves over $\mathbb{P}^{2}$ :

$$
0 \rightarrow \bigoplus_{i=1}^{n-1} \mathcal{O}_{\mathbb{P}^{2}}\left[-l_{i}\right] \stackrel{\psi}{\rightarrow} \bigoplus_{i=1}^{n} \mathcal{O}_{\mathbb{P}^{2}}\left[-k_{i}\right] \stackrel{\gamma}{\rightarrow} \mathcal{O}_{\mathbb{P}^{2}} \rightarrow \mathcal{O}_{Z} \rightarrow 0
$$

By tensoring this complex with $\mathcal{O}_{\mathbb{P}^{2}}(t)$ for $t \gg 0$, all the sheaves of the new complex have no higher cohomology (see 20] III, theorem 5.2). We deduce that the complex obtained by taking the global sections is also exact and so we can compute $\operatorname{dim}_{\mathbb{K}} H^{0}\left(Z, \mathcal{O}_{Z}\right)=\operatorname{dim}_{\mathbb{K}}(R / G)_{t}$ for $t \gg 0$ from the resolution (2) of the ideal $G$.

Notice that in the case where $G$ is a complete intersection, we recover the same results as in [四], that is $N_{i}=\frac{d_{0} d_{1} d_{2}}{d_{i}}-$ $k_{1} k_{2}$, for $i=0,1,2$.

\subsection{The residual of a codimension 2 ideal}

Let $A=\mathbb{K}\left[x_{0}, \ldots, x_{m}\right]$ be a polynomial ring with $m \geq 2$, and $I=\left(g_{1}, \ldots, g_{n}\right)$ be a homogeneous ideal of $A$ such that $A / I$ has projective dimension 2 . We denote by $k_{1} \geq \ldots \geq k_{n}$ the respective degrees of the polynomials $g_{1}, \ldots, g_{n}$. We consider the $s+1$ following polynomials of respective degrees $d_{0} \geq \ldots \geq d_{s} \geq k_{1}$ defined by :

$$
\left\{\begin{aligned}
f_{0}(x) & =\sum_{i=1}^{n} h_{i, 0}(x) g_{i}(x) \\
\vdots & \\
f_{s}(x) & =\sum_{i=1}^{n} h_{i, m}(x) g_{i}(x)
\end{aligned}\right.
$$

where $h_{i, j}=\sum_{|\alpha|=d_{j}-k_{i}} c_{\alpha}^{i, j} x^{\alpha}$ are generic homogeneous polynomials of degree $d_{j}-k_{i}$. We denote by $A_{0}$ the polynomial ring of the parameters $\mathbf{c}=\left(c_{\alpha}^{i, j}\right)$, that is $A_{0}=\mathbb{K}[\mathbf{c}]$, and by $A^{g}$ the polynomial ring $A_{0}\left[x_{0}, \ldots, x_{m}\right]$. We will consider $A^{g}$ as a graded $A_{0}$-algebra, that is we set $\operatorname{deg}\left(c_{\alpha}^{i, j}\right)=0$ and $\operatorname{deg}\left(x_{i}\right)=1$. We denote also by $J \subset I \subset A^{g}$ the homogeneous ideal of $A^{g}$ generated by $f_{0}, \ldots, f_{s}$.

In this section we will give a resolution of the quotient ideal $(J: I)$. This resolution is extracted from a course of Marc Chardin on residual intersections. We will use this resolution in the context of the preceding section to compute a resolution of the ideal $(F: G)$ of $R$ and, by the way, to compute the residual resultant. Before giving the resolution, we justify our interest in ideals of projective dimension 1 by the following property : an ideal $P \subset R=\mathbb{K}\left[x_{0}, x_{1}, x_{2}\right]$ associated to points in $\mathbb{P}^{2}$, that is a saturated homogeneous ideal of codimension 2 of $R$, has projective dimension 1 . In fact, more generally, every ideal $Q$ of $A$ of codimension 2 such that $A / Q$ is Cohen-Macaulay has projective dimension 1 (see 15 theorem 20.15).

The Hilbert-Burch theorem tells us that every minimal graded resolution of $A$-modules of the ideal $I$ is of the form (see [15], theorem 20.15) :

$$
0 \rightarrow \bigoplus_{i=1}^{n-1} A\left[-l_{i}\right] \stackrel{\psi}{\longrightarrow} \bigoplus_{i=1}^{n} A\left[-k_{i}\right] \stackrel{\gamma=\left(g_{1}, \ldots, g_{n}\right)}{\longrightarrow} I \rightarrow 0
$$


with $\sum_{i=1}^{n-1} l_{i}=\sum_{i=1}^{n} k_{i}$. We deduce the following graded presentation of $A^{g}$-modules for the ideal $I / J$ :

$$
\bigoplus_{i=1}^{n-1} A^{g}\left[-l_{i}\right] \bigoplus_{i=0}^{s} A^{g}\left[-d_{i}\right] \stackrel{\psi \oplus \phi}{\longrightarrow} \bigoplus_{i=1}^{n} A^{g}\left[-k_{i}\right] \stackrel{\gamma}{\rightarrow} I / J \rightarrow 0
$$

where $\phi$ is the matrix $\left(h_{i, j}\right)_{1 \leq i \leq n, 0 \leq j \leq s}$.

We remark that we have :

$$
A n n_{A^{g}}(\operatorname{coker}(\psi \oplus \phi))=A n n_{A^{g}}(I / J)=(J: I),
$$

and we recall the following theorem of Buchsbaum-Eisenbud (see [2]) :

Proposition 2.5. Let $S$ be a noetherian ring and $\alpha: S^{m} \rightarrow$ $S^{n}$ be a morphism with $m \geq n$ then

$$
A n n_{S}(\operatorname{coker}(\alpha))^{n} \subseteq I_{n}(\alpha) \subseteq \operatorname{Ann_{S}}(\operatorname{coker}(\alpha)),
$$

where $I_{n}(\alpha)$ denotes the ideal generated by the maximal minors of the matrix of $\alpha$. Moreover, if grade $\left(I_{n}(\alpha)\right)=m-$ $n+1$, then $I_{n}(\alpha)=A n n_{S}(\operatorname{coker}(\alpha))$.

Now the ideal $(J: I)$ is an $s+1$-residual intersection of $I$ in $A^{g}$, that is $\operatorname{codim}(J: I) \geq s+1$ (see 22). Since $A^{g}$ is Cohen-Macaulay we have $\operatorname{codim}(J: I)=\operatorname{grade}(J: I)$, hence $\operatorname{grade}(J: I) \geq s+1=(n+s)-(n)+1$. By the preceeding proposition we conclude that $I_{n}(\psi \oplus \phi)=(J: I)$. From this, we obtain a resolution of the ideal $(J: I)$ by taking the Eagon-Northcott complex $\mathcal{D}_{0}^{g}(\psi \oplus \phi)$. This graded complex is defined as follows (see [1] 2.C for more details or [23]) : Let $M$ and $N$ be the two free graded $A^{g}$-modules defined by

$$
M=\bigoplus_{i=1}^{n-1} A^{g}\left[-l_{i}\right] \bigoplus_{i=0}^{s} A^{g}\left[-d_{i}\right] \stackrel{\psi \oplus \phi}{\longrightarrow} N=\bigoplus_{i=1}^{n} A^{g}\left[-k_{i}\right] .
$$

The complex $\mathcal{D}_{0}^{g}(\psi \oplus \phi)$ is

$$
0 \rightarrow P_{s+1} \stackrel{d f}{\longrightarrow} \ldots \stackrel{d f}{\longrightarrow} P_{i} \stackrel{d f}{\longrightarrow} P_{i-1} \stackrel{d f}{\longrightarrow} \ldots \stackrel{d f}{\longrightarrow} P_{1} \stackrel{\sigma}{\longrightarrow} A^{g}
$$

where $P_{i}=S_{i-1}\left(N^{*}\right) \otimes \bigwedge^{n-1+i}(M), S_{\bullet}$ denotes the symmetric algebra, $\Lambda^{\bullet}$ the exterior algebra and ${ }^{*}$ the dual module. The map $\sigma$ is the map $\wedge^{n}(\psi \oplus \phi)$ and the maps $d f$ are defined as follow : Let $e_{1}, \ldots, e_{n+s}$ be a base of $M$ and $b_{1}, \ldots, b_{n}$ be a base of $N$, then we have

$$
d f\left(\left(b_{i_{1}}^{*} \ldots b_{i_{r}}^{*}\right) \otimes\left(e_{j_{1}} \wedge \ldots \wedge e_{j_{u}}\right)\right)=\sum_{t=1}^{r} \sum_{v=1}^{u}(-1)^{v+1}
$$

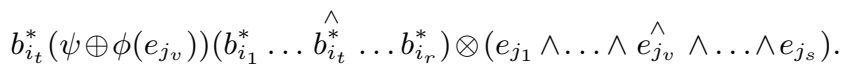

Proposition 2.6. The complex $\mathcal{D}_{0}^{g}(\psi \oplus \phi)$ is a free graded resolution of $A^{g}$-modules of the residual ideal $(J: I)$. This ideal is $\nu_{\mathbf{d}, \mathbf{k}}$-regular with

$$
\nu_{\mathbf{d}, \mathbf{k}}=d_{0}+\ldots+d_{s}-s\left(k_{n}+1\right) .
$$

Moreover, if we specialize all the coefficients $\mathbf{c}$ in $\mathbb{K}$, the specialized complex $\mathcal{D}_{0}(\psi \oplus \phi)$ of $\mathcal{D}_{0}^{g}(\psi \oplus \phi)$ is a free graded resolution of $A$-modules of the specialized ideal $(J: I) \subset A$ if this ideal is an $s+1$-residual intersection in $A$. In this case $(J: I)$ is also $\nu_{\mathbf{d}, \mathbf{k}}$-regular.
Proof. First in the generic case (i.e in $A^{g}$ ) we have seen that $(J: I)$ is a $s+1$-residual intersection implies that $I_{n}(\psi \oplus$ $\phi)=(J: I)$ has grade $(n+s)-(n)+1$. The Eagon-Northcott complex $\mathcal{D}_{0}^{g}(\psi \oplus \phi)$ is hence a resolution of $(J: I)$ (see [1], theorem 2.16). An easy computation from the description of the complex $\mathcal{D}_{0}^{g}(\psi \oplus \phi)$ gives the integer $\nu_{\mathbf{d}, \mathbf{k}}$.

In the specialized case, we remark that the presentation (3) always gives a presentation over $A$ and so, if we suppose that $(J: I)$ is a $s+1$-residual intersection in $A$, we obtain that $I_{n}(\psi \oplus \phi)=(J: I)$ has grade $(n+s)-(n)+1$. Similarly the Eagon-Northcott complex $\mathcal{D}_{0}(\psi \oplus \phi)$ of $A$-modules is hence a resolution of $(J: I)$ and the same computation that in the generic case gives the same integer $\nu_{\mathbf{d}, \mathbf{k}}$ for a bound of the regularity of the ideal $(J: I)$ of $A$ (the regularity do not change by specialization of the coefficients $\mathbf{c})$.

We denote by $\mathbf{M}_{\psi \oplus \phi}^{g}$ the matrix of the map $\psi \oplus \phi$ in the generic case, that is in (3). Let $\Delta_{i_{1}, \ldots, i_{n}}$ be the determinant of the $n \times n$ submatrix of $\mathrm{M}_{\psi \oplus \phi}^{g}$ corresponding to the columns $i_{1}, \ldots, i_{n}$. Then the last map $\sigma$ of the complex $\mathcal{D}_{0}^{g}(\psi \oplus \phi)$ is :

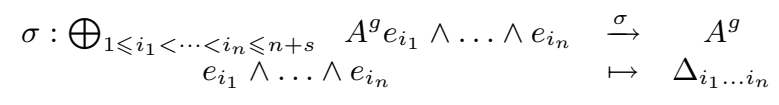

If we denote by $\alpha_{i_{1}, \ldots, i_{n}}$ the degree of the polynomial $\Delta_{i_{1}, \ldots, i_{n}}$, then the preceeding map $\sigma$ in degree $\nu$, denoted $\sigma_{\nu}$, is :

$$
\sigma_{\nu}: \bigoplus A^{g}{ }_{\nu-\alpha_{i_{1}, \ldots, i_{n}}} e_{i_{1}} \wedge \ldots \wedge e_{i_{n}} \rightarrow A^{g}{ }_{\nu}
$$

where $A_{t}^{g}$ denotes the homogeneous part of degree $t$ of the graded $A_{0}$-algebra $A^{g}$. We will denote by $\mathrm{M}_{\nu}^{g}$ its matrix in the monomial bases.

\subsection{Computing the residual resultant}

We return to the situation of section 2.1 in order to compute explicitly the residual resultant using the results of section 2.2. $R$ denotes the polynomial ring $\mathbb{K}\left[x_{0}, x_{1}, x_{2}\right]$. We suppose that $G$ is a homogeneous ideal of $R$ generated by $n$ homogeneous polynomials $g_{1}, \ldots, g_{n}$ of respective degrees $k_{1} \geq \cdots \geq k_{n}$, and that $G$ is a saturated local complete intersection of codimension 2. Let $f_{0}, f_{1}, f_{2}$ be three homogeneous polynomials as defined in (11), of respective degrees $d_{0}, d_{1}, d_{2}$, such that $d_{0} \geq d_{1} \geq d_{2} \geq k_{1}$ and $d_{2} \geq k_{n}+1$. We denote by $A=\mathbb{K}[\mathbf{c}]$, by $R^{g}$ the polynomial $\operatorname{ring} R^{g}=A\left[x_{0}, x_{1}, x_{2}\right]$ and by $F$ the homogeneous ideal of $R^{g}$ generated by the three polynomials $f_{0}, f_{1}, f_{2}$. We will say that we specialized the polynomials $f_{0}, f_{1}, f_{2}$ in $\mathbb{K}$ if we specialized all the coefficients $\mathbf{c}$ in $\mathbb{K}$. In this case the ideal $F$ becomes an ideal of the ring $R$.

Proposition 2.7. Suppose that the polynomials $f_{0}, f_{1}$ and $f_{2}$ are specialized in $\mathbb{K}$, then the following statements are equivalent:

- $\operatorname{Res} \mathcal{G}_{,} d_{0}, d_{1}, d_{2}\left(f_{0}, f_{1}, f_{2}\right) \neq 0$,

- The map $\sigma_{\nu}$ of the specialized complex $\mathcal{D}_{0}(\psi \oplus \phi)$ in degree $\nu$ is surjective for $\nu \geq \nu_{\mathbf{d}, \mathbf{k}}$,

- $V(F: G)=\emptyset$,

- $F^{\text {sat }}=G$. 
Proof. By proposition 2.1 and remark 2.2, it only remains to prove that the map $\sigma_{\nu}$ is surjective for $\nu \geq \nu_{\mathbf{d}, \mathbf{k}}$ if and only if the variety $V(F: G)$ is empty. If $V(F: G)$ is empty, then $(F: G)$ is a 3-residual intersection in $R$ and the image of $\sigma_{\nu}$ is $(F: G)_{\nu}=R_{\nu}$ since $\nu$ is greater or equal to the regularity of $(F: G)$.

Now suppose that $V(F: G)$ is not empty. Then there exists a point $\zeta \in \mathbb{P}^{2}$ such that all the determinants of the maximal minors $\Delta_{i_{1}, \ldots, i_{n}}$ of $\mathrm{M}_{\psi \oplus \phi}$ (the specialized matrix of the matrix $\mathrm{M}_{\psi \oplus \phi}^{g}$ ) vanish. Indeed, we have the following equality :

$$
\left(0, \ldots, 0, f_{0}, f_{1}, f_{2}\right)=\left(g_{1}, \ldots, g_{n}\right) \cdot \mathrm{M}_{\psi \oplus \phi},
$$

and by Cramer's rules all the $\Delta_{i_{1}, \ldots, i_{n}}$ are in the quotient ideal $(F: G)$ (notice that the polynomials $f_{0}, f_{1}, f_{2}$ appear as such minors). We deduce that any polynomial in the image of $\sigma_{\nu}\left(\nu \geq \nu_{\mathbf{d}, \mathbf{k}}\right)$ vanishes at $\zeta$ and hence $\sigma_{\nu}$ is not surjective.

Hereafter $\nu$ is always supposed to be an integer greater or equal to $\nu_{\mathbf{d}, \mathbf{k}}=d_{0}+d_{1}+d_{2}-2\left(k_{n}+1\right)$.

Proposition 2.8. Any nonzero maximal minor of size $\operatorname{rank}\left(R_{\nu}^{g}\right)$ of the matrix $\mathrm{M}_{\nu}^{g}$ (see section 2.2) is a multihomogeneous polynomial in the coefficients $\mathbf{c}$ of $f_{0}, f_{1}, f_{2}$, and a multiple of $\operatorname{Res}_{\mathcal{G}, d_{0}, d_{1}, d_{2}}$.

Proof. Let $\rho$ be a nonzero maximal minor of $\mathrm{M}_{\nu}^{g}$. It is clear that $\rho$ is a homogeneous polynomial in the coefficients of each $f_{i}$ for $i=0,1,2$.

We recall that $X=\mathbb{P}^{2}, \tilde{X}$ is the blow-up of $X$ along the sheaf of ideals associated to $G$ and that $\operatorname{Res}_{\mathcal{G}, d_{0}, d_{1}, d_{2}}$ vanishes if and only if there exists a point $x \in \tilde{X}$ such that the global section $\pi^{*}\left(f_{i}\right)$ of $\tilde{\mathcal{G}}_{d_{i}}$ vanishes at $x$ simultaneously for all $i=$ $0,1,2$ (see proposition 2.1). Let $\tilde{X}^{0}$ be the dense open subset of $\tilde{X}$ defined as the complement of the exceptional divisor in $\tilde{X}$, and let $Z^{0}=\left\{\mathbf{c} \in \prod_{i=0}^{2} V_{i}: \exists x \in \tilde{X}^{0} \pi^{*}\left(f_{0}\right)(x)=\right.$ $\left.\ldots=\pi^{*}\left(f_{2}\right)(x)=0\right\}$. Assume that there exists $\mathbf{c}_{0} \in Z^{0}$ such that $\rho\left(\mathbf{c}_{0}\right) \neq 0$. For this specialization $\mathbf{c}_{0}, \sigma_{\nu}$ is then surjective, which contradicts proposition 2.7. We deduce that $\rho$ vanishes on $Z^{0}$. As $\tilde{X}^{0}$ is dense in $\tilde{X}, Z^{0}$ is also dense in $Z=\left\{\mathbf{c} \in \prod_{i=0}^{2} V_{i}: \exists x \in \tilde{X} \pi^{*}\left(f_{0}\right)(x)=\ldots=\right.$ $\left.\pi^{*}\left(f_{2}\right)(x)=0\right\}$ and consequently $\rho$ vanishes on $Z$, that is $\rho$ is a multiple of $\operatorname{Res} \mathcal{G}, d_{0}, d_{1}, d_{2}$.

Proposition 2.9. For any $i=0,1,2$, there exists a nonzero maximal minor of size $\operatorname{rank}\left(R_{\nu}^{g}\right)$ of $\mathrm{M}_{\nu}^{g}$ of degree $N_{i}$ (see proposition 2.3 and 2.4) in the coefficients of $f_{i}$.

Proof. We choose a specialization of the polynomials $f_{0}, f_{1}, f_{2}$ such that $(F: G)$ is a 3-residual intersection in $R$. Let us denote by $F^{\prime}$ the ideal $\left(f_{1}, f_{2}\right)$. By hypothesis $\left(F^{\prime}: G\right)$ is a 2-residual intersection and its degree is $N_{0}$ by proposition 2.3. Proposition 2.6 tells us that $\left(F^{\prime}: G\right)$ is $\nu^{\prime}$-regular with

$$
\nu^{\prime}=d_{1}+d_{2}-\left(k_{n}+1\right)=\nu_{\mathbf{d}, \mathbf{k}}-d_{0}+k_{n}+1 \leq \nu_{\mathbf{d}, \mathbf{k}}
$$

(by hypothesis $d_{i} \geq k_{n}+1, i=0, \ldots, 2$ ). Since the CastelnuovoMumford regularity bounds the regularity of the Hilbert function (for ideal of dimension zero), for any $s \geq \nu^{\prime}$, we have $\operatorname{dim}_{\mathbb{K}}\left(F^{\prime}: G\right)_{s}=\operatorname{dim}_{\mathbb{K}}\left(R_{s}\right)-N_{0}$.

Consider now the ideal $(F: G)$ in degree $\nu$. It is generated by the multiples of degree $\nu$ of the maximal minors of $\mathrm{M}_{\psi \oplus \phi}$. The last equation implies that the multiples of degree $\nu$ of the maximal minors involving only the coefficients of polynomials $f_{1}$ and $f_{2}$ generate a vector subspace $L$ of dimension $\operatorname{dim}_{\mathbb{K}}\left(R_{\nu}\right)-N_{0}$ of $R_{\nu}$. As $(F: G)_{\nu}=R_{\nu}$, we can complete a basis of $R_{\nu}$ by $N_{0}$ multiples of degree $\nu$ of maximal minors of $\mathrm{M}_{\psi \oplus \phi}$ involving the only column depending on the coefficients of $f_{0}$. Since such minors are of degree 1 in the coefficients of $f_{0}$, we deduce that there exists a maximal minor of $\mathrm{M}_{\nu}$, and hence of $\mathrm{M}_{\nu}^{g}$, of degree $N_{0}$ in the coefficients of $f_{0}$. A similar proof applies by symmetry for $i=1$ and 2 .

TheOREM 2.10. The gcd of all the determinants of the maximal minors of size $\operatorname{rank}\left(R_{\nu}^{g}\right)$ of the matrix $\mathrm{M}_{\nu}^{g}$ is exactly $\operatorname{Res}_{\mathcal{G}, d_{0}, d_{1}, d_{2}}$.

Proof. By proposition 2.8, the gcd of the maximal minors of $\mathrm{M}_{\nu}^{g}$ is divisible by $\operatorname{Res}_{\mathcal{G}, d_{0}, d_{1}, d_{2}}$ which is multihomogeneous of degree $N_{i}$ in the coefficients of the polynomial $f_{i}$. Now by proposition 2.9 there exists a maximal minor of $\mathrm{M}_{\nu}^{g}$ of degree $N_{i}$ in the coefficients of the polynomial $f_{i}$. We deduce that $\operatorname{Res}_{\mathcal{G}, d_{0}, d_{1}, d_{2}}$ is exactly the gcd of all the maximal minors of the matrix $\mathrm{M}_{\nu}^{g}$.

This theorem gives a first method to compute our residual resultant :

\section{Algorithm 1:}

\section{Compute the matrix $\mathrm{M}_{\nu_{\mathbf{d}, \mathbf{k}}}^{g}$.}

2. Compute a maximal minor $\delta_{i}$ of degree $N_{i}$ in the coefficients of $f_{i}$ for $i=0,1,2$.

3. Return the gcd of $\operatorname{det}\left(\delta_{0}\right), \operatorname{det}\left(\delta_{1}\right)$ and $\operatorname{det}\left(\delta_{2}\right)$.

Notice that the maximal minors $\delta_{i}$ are square matrices of size $\operatorname{rank}\left(R_{\nu}^{g}\right)=\left(\begin{array}{c}\nu+2 \\ 2\end{array}\right)$. In practice, to compute such a maximal minor of $\mathrm{M}_{\nu}^{g}$ of degree $N_{i}$ in the coefficients of the polynomial $f_{i}$, we compute first a maximal minor of the matrix $\mathrm{M}_{\nu}^{\prime g}$ associated to the ideal $\left(\left(f_{1}, f_{2}\right): G\right)$ and complete it incrementally with the right number of columns of $\mathrm{M}_{\nu}^{g}$ depending on the coefficients of $f_{0}$.

We can also compute our residual resultant as the determinant of a complex as for other classical resultants (see 18] appendix A for definition and properties of the determinant of a complex). We denote by $\mathcal{D}_{0}^{g}(\psi \oplus \phi)_{\nu}$ the complex $\mathcal{D}_{0}^{g}(\psi \oplus \phi)$ in degree $\nu$. It is a complex of free $A$-modules of finite type.

TheOREM 2.11. The determinant of the complex $\mathcal{D}_{0}^{g}(\psi \oplus \phi)_{\nu}$ is exactly the residual resultant $\operatorname{Res} \mathcal{G}, d_{0}, d_{1}, d_{2}$ for all $\nu \geq \nu_{\mathbf{d}, \mathbf{k}}$.

Proof. By proposition 2.6 the complex $\mathcal{D}_{0}^{g}(\psi \oplus \phi)_{\nu}$ is generically exact, that is exact after tensoring by the field of fractions of $A$ over $A$, and is acyclic, that is all its homology groups are zero except in degree 0 . Theorem 34 of 18 tells us that its determinant is equal to the gcd of the maximal minors of the map $\sigma_{\nu}$ which is exactly the residual resultant by proposition 2.10 . 
The complex $\mathcal{D}_{0}^{g}(\psi \oplus \phi)_{\nu}$ has only three terms in our case. It is of the form :

$$
\bigoplus_{i=1}^{\frac{(n+1) n}{2}} R_{\nu-\delta_{i}}^{g} \stackrel{d f_{2}}{\longrightarrow} \bigoplus_{i=1}^{n(n+2)} R_{\nu-\beta_{i}}^{g} \stackrel{d f_{1}}{\longrightarrow} \bigoplus_{i=1}^{\frac{(n+1)(n+2)}{2}} R_{\nu-\alpha_{i}}^{g} \stackrel{\sigma}{\longrightarrow} R_{\nu}^{g}
$$

An algorithm for computing the determinant of such a complex of free modules (due to Cayley) can be found in 18 theorem 14, or in [9]. In our case we can compute the residual resultant by the following algorithm . For simplicity, we denote the complex $\mathcal{D}_{0}^{g}(\psi \oplus \phi)_{\nu}$ by

$$
0 \rightarrow L_{3} \stackrel{d f_{2}}{\longrightarrow} L_{2} \stackrel{d f_{1}}{\longrightarrow} L_{1} \stackrel{\sigma}{\rightarrow} L_{0},
$$

and by $B_{0}, B_{1}, B_{2}, B_{3}$ respective bases of $L_{0}, L_{1}, L_{2}, L_{3}$. Algorithm 2:

1. Compute a maximal minor $\delta_{0}$ of the map $\sigma: B_{1} \rightarrow B_{0}$. This gives a base $K_{1} \subset B_{1}$ with $\sharp K_{1}=\sharp B_{0}$ (where $\sharp$ denotes the cardinality).

2. Compute a maximal minor $\delta_{1}$ of the map $d f_{1}: B_{2} \rightarrow$ $B_{1} \backslash K_{1}$. This gives a base $K_{2} \subset B_{2}$ with $\sharp\left(K_{2}\right)=$ $\sharp\left(B_{1} \backslash K_{1}\right)$.

3. Return the residual resultant wich is $\frac{\operatorname{det}\left(\delta_{0}\right) \operatorname{det}\left(\delta_{2}\right)}{\operatorname{det}\left(\delta_{1}\right)}$, where $\delta_{2}$ is the matrix of the map $d f_{2}: B_{3} \rightarrow B_{2} \backslash K_{2}$.

We illustrate our construction with one example. A package, called multires, implements this residual resultant formulation among other more classical resultant matrix constructions. It is available at

http://www.inria.fr/saga/logiciels/multires

We consider the ideal $G=\left(x_{0} x_{1}, x_{0} x_{2}, x_{1} x_{2}\right)$ of $R$. This ideal defines three smooth points of $\mathbb{P}^{2}$. Its resolution is :

$$
0 \rightarrow \bigoplus_{i=1}^{2} R[-3] \stackrel{\psi}{\rightarrow} \bigoplus_{i=1}^{3} R[-2] \stackrel{\gamma}{\rightarrow} G \rightarrow 0
$$

with, $\psi=\left(\begin{array}{cc}-x_{2} & 0 \\ x_{1} & -x_{1} \\ 0 & x_{0}\end{array}\right), \quad \gamma=\left(x_{0} x_{1}, x_{0} x_{2}, x_{1} x_{2}\right)$.

We suppose first that the $h_{i, j}(x)=c_{i, j}^{0} x_{0}+c_{i, j}^{1} x_{1}+c_{i, j}^{2} x_{2}$ are linear forms of $R_{1}^{g}$. We find $\nu_{\mathbf{d}, \mathbf{k}}=3$ and $N_{0}=N_{1}=$ $N_{2}=6$. The matrix $\mathrm{M}_{3}^{g}$ is a $10 \times 10$ matrix and its determinant is exactly the residual resultant. In this case the complex $\mathcal{D}_{0}^{g}(\psi \oplus \phi)_{3}$ degenerates into the isomorphism $\mathrm{M}_{3}^{g}: \bigoplus_{i=1}^{10} R_{0}^{g} \rightarrow R_{3}^{g}$.

If now we suppose that $h_{i, 2}$ are generic forms of degree 2, then $\nu_{\mathbf{d}, \mathbf{k}}=4, N_{0}=N_{1}=9$ and $N_{2}=6$. In this case $\mathrm{M}_{4}^{g}$ is a $15 \times 18$ matrix and the residual resultant is given by a quotient of two determinants.

Before ending this section we mention the case where the ideal $G$ is a complete intersection of codimension two. In this case our residual resultant corresponds to the residual resultant constructed in [4, and the matrix which computes it is exactly $\mathbf{M}_{\nu_{\mathbf{d}, \mathbf{k}}}^{g}$.

\section{IMPLICITIZATION OF RATIONAL SUR- FACES}

We consider four homogeneous polynomials $p_{0}\left(x_{0}, x_{1}, x_{2}\right)$, $p_{1}\left(x_{0}, x_{1}, x_{2}\right), p_{2}\left(x_{0}, x_{1}, x_{2}\right)$ and $p_{3}\left(x_{0}, x_{1}, x_{2}\right)$ of the same degree $d$ of the polynomial ring $R=\mathbb{K}\left[x_{0}, x_{1}, x_{2}\right]$. The equations :

$$
x=\frac{p_{0}\left(x_{0}, x_{1}, x_{2}\right)}{p_{3}\left(x_{0}, x_{1}, x_{2}\right)}, y=\frac{p_{1}\left(x_{0}, x_{1}, x_{2}\right)}{p_{3}\left(x_{0}, x_{1}, x_{2}\right)}, z=\frac{p_{2}\left(x_{0}, x_{1}, x_{2}\right)}{p_{3}\left(x_{0}, x_{1}, x_{2}\right)} .
$$

define a parametrization of a rational surface. The implicitization problem consists in finding the polynomial $H(x, y, z)$ of smallest degree such that $H(x, y, z)=0$.

We will call a base point of the rational surface a triple $(a, b, c)$ such that $p_{i}(a, b, c)=0$ for $i=0, \ldots, 3$. In the abscence of base points a classical method for finding the implicit equation is to compute the projective resultant over $\mathbb{P}^{2}=\operatorname{Proj}(R)$ of the polynomials $p_{0}-x p_{3}, p_{1}-y p_{3}, p_{2}-z p_{3}$. This projective resultant is exactly $H(x, y, z)^{m}$ where $m$ is the degree of the parametrization map of the surface (see 11] for more details). In the presence of base points the preceding projective resultant is degenerated. Under some conditions on the degree of the generators of the base points locus ideal, we will see that we can use the residual resultant to remove these base points and hence to reduce the problem of finding the implicit equation to computing a residual resultant.

As we have remarked, the base points locus of codimension 1 is easily removed by a gcd computation. Consequently we will concentrate on the base points locus of codimension 2 .

\subsection{Rational surface with local complete inter- section base points locus}

Let $G=\left(g_{1}, \ldots, g_{n}\right)$ be a homogeneous ideal of $R$ of codimension 2 defining the base point locus (that is saturated), where $g_{1}, \ldots, g_{n}$ are homogeneous polynomials of respective degrees $k_{1} \geq \ldots \geq k_{n}$. This implies

$$
\left(p_{0}, p_{1}, p_{2}, p_{3}\right)^{s a t}=G .
$$

In order to apply the results of the preceding sections, we suppose that $G$ is a local complete intersection ideal of codimension 2 and hence admits the minimal resolution (2). Let $\mathcal{G}$ be the ideal sheaf of $\mathbb{P}^{2}$ associated to the ideal $G$. The sheaf $\mathcal{G}(d)$ is generated by its global sections $p_{0}, p_{1}, p_{2}$ and $p_{3}$. We denote by $\pi: \tilde{\mathbb{P}}^{2} \rightarrow \mathbb{P}^{2}$ the blow up of $\mathbb{P}^{2}$ along $\mathcal{G}$, and by $\tilde{p}_{i}$ the global section $\pi^{*}\left(p_{i}\right)$ of the sheaf $\tilde{\mathcal{G}}_{d}$ for $i=0, \ldots, 3$ (see proof of proposition 2.1). Since $\tilde{\mathcal{G}}_{d}$ is an invertible sheaf on $\tilde{\mathbb{P}}^{2}$ and $\tilde{p}_{0}, \tilde{p}_{1}, \tilde{p}_{2}, \tilde{p}_{3}$ are global sections which generate it, we deduce that there exists a morphism

$$
\theta: \tilde{\mathbb{P}}^{2} \longrightarrow \mathbb{P}^{3}=\operatorname{Proj}(\mathbb{K}[x, y, z, w])
$$

such that $\theta^{*} \mathcal{O}(1) \cong \tilde{\mathcal{G}}_{d}$ and $\theta^{*}(x)=\tilde{p}_{0}, \theta^{*}(y)=\tilde{p}_{1}, \theta^{*}(z)=$ $\tilde{p}_{2}, \theta^{*}(w)=\tilde{p}_{3}$ (see [20], II.7). As $\tilde{\mathbb{P}}^{2}$ is projective and irreducible, we have $\theta_{*}\left(\widetilde{\mathbb{P}}^{2}\right)=\operatorname{deg}\left(\tilde{\mathbb{P}}^{2} / S\right) S$ where $S=\theta\left(\tilde{\mathbb{P}}^{2}\right)$ is our rational surface in $\mathbb{P}^{3}$ and $\operatorname{deg}\left(\tilde{\mathbb{P}}^{2} / S\right)$ denotes the degree of the surjective map $\theta: \tilde{\mathbb{P}}^{2} \rightarrow S$.

Let $\beta$ be the following regular map :

$$
\begin{aligned}
& \beta: \quad U=\mathbb{P}^{2} \backslash V(G) \longrightarrow \mathbb{P}^{3} \\
& \left(x_{0}: x_{1}: x_{2}\right) \quad \mapsto \quad\left(p_{0}: p_{1}: p_{2}: p_{3}\right)
\end{aligned}
$$


Proposition 3.1. The degree of $S$ divides

$$
d^{2}-\frac{\sum_{i=1}^{n-1} l_{i}^{2}-\sum_{i=1}^{n} k_{i}^{2}}{2}
$$

(where the integers $k_{i}$ and $l_{i}$ are defined by (19)) and is equal to it when $\beta$ is birational.

Proof. We have $\operatorname{deg}\left(\theta_{*}\left(\tilde{\mathbb{P}}^{2}\right)\right)=\operatorname{deg}\left(\tilde{\mathbb{P}}^{2} / S\right) \operatorname{deg}(S)$ and

$$
\operatorname{deg}\left(\theta_{*}\left(\tilde{\mathbb{P}}^{2}\right)\right)=\int_{\tilde{\mathbb{P}}^{2}} c_{1}\left(\theta^{*}(\mathcal{O}(1))\right)^{2}=\int_{\tilde{\mathbb{P}}^{2}} c_{1}\left(\tilde{\mathcal{G}}_{n}\right)^{2},
$$

which is exactly $d^{2}-\frac{\sum_{i=1}^{n-1} l_{i}^{2}-\sum_{i=1}^{n} k_{i}^{2}}{2}$ by propositions 2.3 and 2.4.

Now we consider the following diagram where $E$ denotes the exceptionnal divisor of the blow-up $\pi$ :

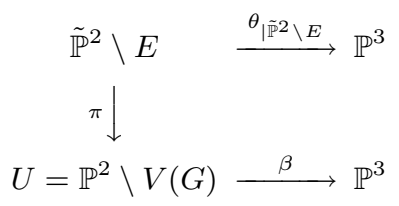

Since by construction $\theta$ is unique and since the vertical map is an isomorphism (the blow-up is an isomorphism outside the exceptional divisor), we deduce that $\theta_{\mid \tilde{\mathbb{P}}^{2} \backslash E}=\beta \circ \pi$, and hence $\operatorname{deg}\left(\tilde{\mathbb{P}}^{2} / S\right)=\operatorname{deg}(U / \beta(U))$ which is 1 if $\pi$ is birational.

The equation $S=0$ is the homogeneous implicit equation of our rational surface. We have $H(x, y, z)=S(x, y, z, 1)$ and $\operatorname{deg}(S)=\operatorname{deg}(H)$. We can compute explicitly $H$ by computing a residual resultant :

Theorem 3.2. Suppose that $d \geq k_{1} \geq \ldots \geq k_{n}$, that $d \geq$ $k_{n}+1$ and that $\left(p_{0}, p_{1}, p_{2}, p_{3}\right)^{\text {sat }}=G$, then

$\operatorname{Res}_{\mathcal{G}, d, d, d}\left(p_{0}-x p_{3}, p_{1}-y p_{3}, p_{2}-z p_{3}\right)=H(x, y, z)^{\operatorname{deg}(U / \beta(U))}$, with $\operatorname{deg}(U / \beta(U))=1$ if $\beta$ is birational.

Proof. The residual resultant is defined as a general resultant over the blow-up of $\mathbb{P}^{2}$ along $\mathcal{G}$ (see [象). Let $t$ denote a point of $\tilde{\mathbb{P}}^{2} \backslash V\left(\tilde{p}_{3}=0\right)$ and $\tilde{\mathcal{W}}$ the variety

$\left\{t \times(x, y, z): \tilde{p}_{0}(t)-x \tilde{p}_{3}(t)=\tilde{p}_{1}(t)-y \tilde{p}_{3}(t)=\tilde{p}_{2}(t)-z \tilde{p}_{3}(t)=0\right\}$, we have the following diagram :

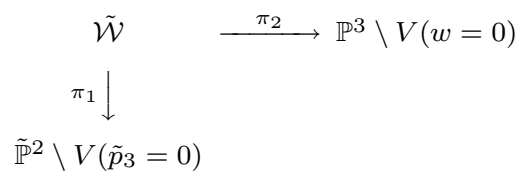

The residual resultant $\operatorname{Res}_{\mathcal{G}, d, d, d}\left(p_{0}-x p_{3}, p_{1}-y p_{3}, p_{2}-z p_{3}\right)$ is exactly $\pi_{2 *}(\tilde{\mathcal{W}})$, and as the blow-up is an isomorphism outside the exceptional divisor, this residual resultant vanishes if and only if the affine point $(x, y, z)$ of $\mathbb{P}^{3}$ is on $H$. We deduce that

$\operatorname{Res}_{\mathcal{G}, d, d, d}\left(p_{0}-x p_{3}, p_{1}-y p_{3}, p_{2}-z p_{3}\right)=H^{\operatorname{deg}\left(\tilde{\mathcal{W}} / \pi_{2}(\tilde{\mathcal{W}})\right)}$.

Now the map $\beta_{\mid V\left(\tilde{p}_{3} \neq 0\right)}$ makes the preceding diagram commutative, and since $\pi_{1}$ is birational we deduce that $\operatorname{deg}\left(\tilde{\mathcal{W}} / \pi_{2}(\tilde{\mathcal{W}})\right)=\operatorname{deg}(U / \beta(U))$.

\subsection{Computing the implicit equation}

Theorem 3.2 shows that we can compute the implicit equation $H(x, y, z)$ of a rational surface as a residual resultant if the base points locus ideal $G$ is a local complete intersection, $d \geq k_{1} \geq \ldots \geq k_{n}$ and $d \geq k_{n}+1$.

REMARK 3.3. Smooth (or reduced) points are always a local complete intersection, so we can always compute the implicit equation of a rational surface with smooth base points locus by computing a residual resultant provided that $d \geq k_{1}$ and $d \geq k_{n}+1$.

To compute explicitly the implicit equation we use algorithms 1 and 2. To do this, we recall that the ideal $G$ has the resolution (2). As $\left(p_{0}, p_{1}, p_{2}, p_{3}\right) \subset G$, there exists a $n \times 4$ matrix $Q$ such that $\left(p_{0}, p_{1}, p_{2}, p_{3}\right)=\left(g_{1}, \ldots, g_{n}\right) Q$, and we deduce a matrix $H$ such that :

$$
\left(p_{0}-x p_{3}, p_{1}-y p_{3}, p_{2}-z p_{3}\right)=\left(g_{1}, \ldots, g_{n}\right) H .
$$

This matrix $H$ defines the map

$$
\bigoplus_{i=1}^{3} R^{g}\left[-d_{i}\right] \stackrel{\phi}{\rightarrow} \bigoplus_{i=1}^{n} R^{g}\left[-k_{i}\right]
$$

where $R^{g}$ is the graded $\mathbb{K}[x, y, z]$-algebra $\mathbb{K}[x, y, z]\left[x_{0}, x_{1}, x_{2}\right]$. We obtain the map $\psi \oplus \phi$ between the two following free $R^{g}$ modules :

$$
M=\bigoplus_{i=1}^{n-1} R^{g}\left[-l_{i}\right] \oplus \bigoplus_{i=0}^{3} R^{g}\left[-d_{i}\right] \stackrel{\psi \oplus \phi}{\longrightarrow} N=\bigoplus_{i=1}^{n} R^{g}\left[-k_{i}\right] .
$$

At this point we are ready to apply both algorithms of section 2.3 for computing exactly the implicit equation of our rational surface as a residual resultant. The first algorithm gives the implicit equation as a gcd of three polynomials. The second algorithm gives the implicit equation as the product of two determinants divided by another one and, in particular cases, the implicit equation appears as a quotient of two determinants. The following proposition gives these particular cases :

Proposition 3.4. If $k_{1}=\ldots=k_{n}$ then the implicit equation of the rational surface $H$ is given by a quotient of two determinants.

Proof. We have seen that the implicit equation $H$ is obtained as the determinant of the complex (4) with $\nu=$ $\nu_{\mathbf{d}, \mathbf{k}}=3 n-2\left(k_{n}+1\right)$. We deduce that if for all $i=$ $1, \ldots, \frac{(n+1) n}{2}, 3 n-2 k_{n}+1-\delta_{i}$ is negative, then the last map of the complex (何) is zero and so its determinant is the quotient of two determinants. The condition given in the proposition implies that $3 n-2 k_{n}+1-\delta_{i} \leq 0$ for all $i=1, \ldots, \frac{(n+1) n}{2}$.

As an example, we consider the following rational surface given by :

$$
p_{0}=x_{0} x_{1}^{2}, p_{1}=x_{1}^{3}, p_{2}=x_{0} x_{2}^{2}, p_{3}=x_{1}^{3}+x_{2}^{3} .
$$

The base point locus is a point of multiplicity 4 given by the ideal $G=\left(x_{1}^{2}, x_{2}^{2}\right)$ which is a complete intersection. The resolution of the ideal $G$ is given by a Koszul complex and we thus deduce the matrix $\mathrm{H}$ :

$$
H=\left(\begin{array}{ccc}
x_{0}-X x_{1} & x_{1}-Y x_{1} & -Z x_{1} \\
-X x_{2} & -Y x_{2} & x_{0}-Z x_{2}
\end{array}\right) .
$$


The matrix $\mathrm{M}_{3}^{g}$ is a $10 \times 12$ matrix. Applying the algorithm 1 , we compute a first maximal minor of it and obtain

$$
\left(\begin{array}{cccccccccc}
0 & 0 & 0 & -X & 1 & 0 & 0 & 1-Y & 0 & 0 \\
X & -Y & 0 & 0 & -Z & -X & 0 & -Z & 0 & 0 \\
0 & 0 & 0 & 0 & 0 & 0 & -X & 0 & -Y & -Z \\
0 & 0 & 0 & 1 & 0 & 0 & 0 & 0 & 0 & 0 \\
0 & 0 & 0 & 0 & 0 & 0 & -X & 0 & 1-Y & -Z \\
0 & 0 & 0 & 0 & -X & 0 & 1 & 0 & 0 & 0 \\
-Y & 0 & 0 & -Z & 0 & 1 & 0 & 0 & 0 & 0 \\
0 & 0 & -Y & 0 & 0 & -Z & 0 & 0 & 0 & 1 \\
0 & X & 0 & 0 & 0 & 0 & 0 & 0 & 0 & 0 \\
0 & 0 & X & 0 & 0 & 0 & 0 & 0 & 0 & 0
\end{array}\right)
$$

Its determinant is $\left(-Z^{3} Y^{2}+Y^{2} X^{3}-2 X^{3} Y+X^{3}\right) X^{2}$. The determinant of a second maximal minor is $(-1+Y) Y\left(-Z^{3} Y^{2}+\right.$ $\left.Y^{2} X^{3}-2 X^{3} Y+X^{3}\right)$, and hence, we deduce that the implicit equation of our rational surface is $-Z^{3} Y^{2}+Y^{2} X^{3}-$ $2 X^{3} Y+X^{3}$. The implicit equation is of degree $9-4=5$, as predicted by proposition 3.1.

Now we apply the second algorithm. First, we choose a maximal minor $\delta_{0}$ of $\mathrm{M}_{3}^{g}$, and obtain a base $K_{1}$ of $B_{1}$. The determinant of $\delta_{0}$ is $\left(-Z^{3} Y^{2}+Y^{2} X^{3}-2 X^{3} Y+X^{3}\right) X^{2}$. By proposition 3.4, $K_{2}$ is empty and thus we do not have any choice for $\delta_{1}$ which is given by the following matrix :

$$
\left(\begin{array}{cc}
-X & 0 \\
0 & -X
\end{array}\right)
$$

We hence deduce that the implicit equation is well $-Z^{3} Y^{2}+$ $Y^{2} X^{3}-2 X^{3} Y+X^{3}$.

\section{ACKNOWLEDGMENTS}

I would like to thank my two thesis supervisors André Hirschowitz and Bernard Mourrain for their help and support. I am also grateful to Carlos D'Andrea, Marc Chardin, David Cox and Mohamed Elkadi for helpful comments and discussions.

\section{REFERENCES}

[1] Bruns, W., And Vetter, U. Determinantal rings. Lecture Notes in Mathematics 1327 (1980).

[2] Buchsbaum, D. A., And Eisenbud, D. What annihilates a module? Journal of Algebra 47 (1977), 231-243.

[3] Busé, L., Elkadi, M., And Mourrain, B. Generalized resultants for unirationnal algebraic varieties. J. of Symbolic Computation 59 (2000), $515-526$.

[4] Busé, L., Elkadi, M., And Mourrain, B. Resultant over the residual of a complete intersection. Journal of Pure and Applied Algebra to appear (2001).

[5] Canny, J. F. Generalised characteristic polynomials. Journal of Symbolic Computation 9 (1990), 241-250.

[6] Canny, J. F., And Manocha, D. A new approach for surface intersection. International Journal of Computational Geometry and Applications 1, 4 (1991), 491-516.

[7] Canny, J. F., And Manocha, D. Algorithm for implicitizing rational parametric surfaces. Computer Aided Geometric Design 9 (1992), 25-50.
[8] Canny, J. F., and Manocha, D. Implicit representation of rational parametric surfaces. Journal of Symbolic Computation 13 (1992), 485-510.

[9] Chardin, M. The resultant via a koszul complex. Computational Algebraic Geometry 109 (1993).

[10] Cox, D., Little, J., And O'Shea, D. Ideals, Varieties, and algorithms. Springer, 1996.

[11] Cox, D., Little, J., And O'Shea, D. Using algebraic geometry. Graduate Texts in Mathematics. Springer, 1998.

[12] Cox, D. A. Equations of parametric curves and surfaces via syzygies. Contemporary Mathematics to appear (2001).

[13] Cox, D. A., Goldman, R., And Ming, Z. On the validity of implicitization by moving quadrics for rationnal surfaces with no base points. J. of Symbolic Computation 29 (2000).

[14] D'Andrea, C. Resultants and moving surfaces. Preprint (2000).

[15] Eisenbud, D. Commutative Algebra with a view toward Algebraic Geometry, vol. 150 of Graduate Texts in Math. Springer-Verlag, 1994.

[16] Elkadi, M., And Mourrain, B. Géométrie algébrique effective : de la théorie aux applications. to appear, 2001.

[17] Fulton, W. Intersection theory. Springer-Verlag, 1984.

[18] Gelfand, I., Kapranov, M., And Zelevinsky, A. Discriminants, Resultants and Multidimensional Determinants. Birkhäuser, Boston-Basel-Berlin, 1994.

[19] Griffiths, P., And Harris, J. Principles of algebraic geometry. Wiley-interscience, London-New-York, 1978.

[20] Hartshorne, R. Algebraic Geometry. Springer-Verlag, 1977.

[21] Hoffmann, C. M. Geometric and solid modeling : an introduction. Morgan Kaufmann publishers, Inc., 1989.

[22] Huneke, C., And UlRich, B. Residual intersections. J. reine angew. Math. 390 (1988), 1-20.

[23] LazARD, D. Algèbre linéaire sur $k\left[x_{1}, \ldots, x_{n}\right]$ et élimination. Bull. Soc. math. France 105 (1977), $165-190$

[24] Sederberg, T., And Chen, F. Implicitization using moving curves and surfaces. Proceedings of SIGGRAPH (1995), 301-308. 